\title{
Levantamento do estado nutricional e de complicações associadas à terapia nutricional enteral domiciliar em idosos
}

\author{
Survey of nutritional status and complications associated to home enteral nutritional therapy \\ in the elderly
}

DOI: $10.37111 /$ braspenj.2020352004

Mariangela da Silva Alves Batista

Fabíola de Souza Silva²

\section{Unitermos:}

Terapia nutricional. Estado nutricional. Assistência domiciliar. Idoso.

\section{Keywords:}

Nutrition therapy. Nutritional status. Home nursing. Aged.

\section{Endereço de correspondência:} Mariangela da Silva Alves Batista Rua Ana Clélia Rodrigues, 384 - Vila Isabel - Osasco, SP, Brasil - CEP 06180-020

E-mail: maribatista.nutri@gmail.com

\section{Submissão}

13 de agosto de 2019

\section{Aceito para publicação}

20 de março de 2020

\begin{abstract}
RESUMO
Introdução: A alta prevalência de doenças crônicas não transmissíveis (DCNT) e doenças neurodegenerativas na população idosa têm aumentando o risco de internações hospitalares e, consequentemente, o risco de desnutrição, o que leva a maior necessidade da terapia nutricional enteral (TNE). Sendo assim, o presente artigo tem como objetivo realizar um levantamento do estado nutricional e das complicações associadas à TNE domiciliar (TNED) em idosos. Método: Trata-se de um estudo de delineamento observacional descritivo, realizado em um município de médio porte da Grande São Paulo (Região Metropolitana). Para coleta dos dados foi realizado um levantamento nos prontuários e visitas domiciliares, no período de janeiro de 2012 a abril de 2018. Foram elegíveis os pacientes com 60 anos ou mais de idade, que tinham informações sobre o hábito intestinal, presença ou não de úlceras de pressão, via de administração da dieta enteral e dados antropométricos da circunferência da panturrilha $(C P)$ e circunferência do braço (CB). Os valores encontrados da CP e CB foram interpretados de acordo com os pontos de corte de referência. Resultados: Participaram da pesquisa 43 idosos, sendo $67,4 \%$ do sexo feminino e $32,6 \%$ do sexo masculino. A via de administração da dieta mais utilizada foi a nasoenteral $(62,8 \%)$. Em relação às complicações associadas à TNED, a úlcera de pressão foi a mais prevalente $(58,1 \%)$. A diarreia esteve presente em $4,7 \%$ dos idosos e a constipação em $44,2 \%$. Quanto ao estado nutricional, $88,4 \%$ dos idosos estavam com depleção de massa muscular e $62,9 \%$ estavam com déficit grave de massa. Conclusão: $O$ levantamento do estado nutricional e das complicações associadas à TNED em idosos permitiu verificar que mais da metade apresenta algum déficit de massa muscular e que a úlcera de pressão foi a complicação mais prevalente, seguida da constipação e diarreia.
\end{abstract}

\section{ABSTRACT}

Introduction: The high prevalence of noncommunicable diseases (NCDs) and neurodegenerative diseases in elderly has been increasing the hospitalizations risk and, consequently, the malnutrition risk, which leads to a greater need for enteral tube feeding (ETF). Thus, this article aims to conduct a survey about nutritional status and complications related to home enteral tube feeding (HETF) in elderly. Methods: This is a descriptive observational study conducted in a medium-sized municipality of Greater São Paulo (Metropolitan region). To collect data, a survey of medical records and home visits was carried out from January 2012 to April 2018. Patients aged 60 years and over who had information on bowel habits, presence of pressure ulcers, position of feeding tube and anthropometric data of calf circumference (CC) and arm circumference (AC) were eligible. The values found for $\mathrm{CC}$ and $\mathrm{AC}$ were interpreted according to the reference cut-off points. Results: The study included 43 elderly people, $67.4 \%$ female and $32.6 \%$ male. The nasogastric feeding tube was present in $62,8 \%$ of the patients. Pressure ulcer was the most prevalent (58.1\%) complications associated with HETF. Diarrhea was present in $4.7 \%$ of the elderly and constipation in $44.2 \%$. In regard of nutritional status, muscle mass depletion was present in $88.4 \%$ of the elderly and severe mass deficit in $62.9 \%$ of them. Conclusions: The assessment of the nutritional status and complications associated with HETF in elderly showed that more than a half had some muscle mass deficit and the pressure ulcer was the most prevalent complication, followed by constipation and diarrhea.

1. Nutricionista, Mestre em Saúde Coletiva, Pós-graduada em Saúde da Família, Docente na Universidade Paulista (UNIP), Alphaville, SP, Brasil.

2. Discente na Universidade Paulista (UNIP), Alphaville, SP, Brasil. 


\section{INTRODUÇÃO}

Nos países em desenvolvimento, como o Brasil, considera-se idoso todo indivíduo com 60 anos ou mais. De acordo com a World Health Organization' ', em 2025, existirá um total de aproximadamente 1,2 bilhões de pessoas com mais de 60 anos, sendo que o Brasil será o sexto país do mundo em número de idosos.

O aumento da expectativa de vida, associado à urbanização e às mudanças econômicas e sociais das últimas décadas, teve grande impacto no modo de viver, trabalhar e se alimentar dos brasileiros, o que, por sua vez, apresenta íntima relação com o padrão de doenças crônicas não transmissíveis (DCNT) da atualidade 2 . As DCNT atingem, principalmente grupos vulneráveis, como os idosos e a população de baixa escolaridade e baixa renda, sendo as doenças cardiovasculares, a hipertensão arterial sistêmica e o diabetes mellitus (DM) apontadas como as principais causas de morbidade, incapacidade e mortalidade em todos os países ${ }^{1,2}$.

Além disso, as doenças neurodegenerativas, como a demência, também apresentam alta prevalência na população idosa, principalmente nos países em desenvolvimento, nos quais este dado é de, aproximadamente, $60 \%$ e estimase que, em 2040, chegue a $71,2 \%^{3}$. A disfagia, geralmente relacionada às doenças neurológicas e cânceres de cabeça/ pescoço e esôfago, afeta cerca de $45 \%$ dos idosos com demência e $68 \%$ dos idosos institucionalizados ${ }^{4}$.

Tal perfil epidemiológico aumenta o risco de internações hospitalares e, consequentemente, o risco de desnutrição, o que leva a uma maior necessidade da terapia nutricional enteral $(T N E)^{5}$.

A TNE é um conjunto de procedimentos terapêuticos cujo objetivo é manter e/ou recuperar o estado nutricional do paciente por meio da nutrição enteral, a qual pode ser administrada via oral ou por sondas ${ }^{6}$. A TNE por sonda é indicada aos pacientes que não conseguem alcançar suas necessidades nutricionais pela alimentação via oral, seja em âmbito hospitalar ou domiciliar?. Em âmbito domiciliar, a TNE é indicada em casos em que há disfagia por doenças neurológicas, cardiovasculares e câncer do trato gastrointestinal ${ }^{8}$.

Estudos indicam que é possível propiciar um ambiente familiar e confortável aos pacientes que estão em terapia nutricional enteral domiciliar (TNED) ${ }^{8}$. No entanto, verificase que, tanto estes, quanto seus familiares encontram dificuldades para a realização dos procedimentos de maneira adequada, o que acarreta em complicações, como diarreia, vômito, úlcera de pressão (UP) e pneumonia, que dificultam a recuperação do paciente e a manutenção do tratamento 9,10.

Uma das ferramentas mais importantes para acompanhar o desenvolvimento nutricional dos pacientes, bem como auxiliar na elaboração da proposta terapêutica, é a avaliação do estado nutricional ${ }^{1}$. No entanto, a medição do peso e altura em pacientes acamados e com dificuldades na deambulação apresenta certas limitações ${ }^{12}$. Nesse sentido, medidas possíveis de aferir, como as circunferências do braço (CB) e panturrilha (CP), têm se mostrado bons indicadores do estado nutricional ${ }^{11}$.

Sendo assim, o presente estudo tem como objetivo realizar um levantamento do estado nutricional e das complicações associadas à TNED em idosos de um município da Grande São Paulo, SP.

\section{MÉTODO}

Trata-se de um estudo de delineamento observacional descritivo, realizado em um município de médio porte (aproximadamente 200.000 habitantes) da Grande São Paulo (Região Metropolitana).

Para coleta dos dados foi feito um levantamento nos prontuários e nos relatórios de visitas domiciliares realizadas pela nutricionista do município. O período avaliado foi de janeiro de 2012 a abril de 2018. Foram considerados os dados da última avaliação. Os pacientes elegíveis para o estudo foram aqueles com 60 anos ou mais, que tinham informações sobre o hábito intestinal, presença ou não de UPs, via de administração da dieta enteral e dados da CP e CB.

Os valores da CB (em centímetros) foram classificados em percentil e interpretados de acordo com Kuczmarski et al. ${ }^{13}$, a saber: $C B$ menor do que o percentil 10 é classificado como déficit grave de massa; $C B$ entre o percentil 10 e o percentil 15 é classificado como déficit leve de massa; $C B$ entre o percentil 15 e o percentil 75 é classificado como massa normal e CB entre o percentil 75 e o percentil 95 é classificado como massa aumentada.

Para interpretação dos dados da CP, foi utilizado o ponto de corte proposto por Bonnefoy et al. ${ }^{14}$, no qual valor menor do que $30,5 \mathrm{~cm}$ caracteriza depleção de massa muscular.

O protocolo da pesquisa foi aprovado, em 13 de agosto de 2018, pelo Comitê de Ética em Pesquisa da Universidade Paulista (UNIP), sob o número 2.817.279.

\section{RESULTADOS}

Foi realizado um levantamento de 56 prontuários e relatórios de visitas domiciliares. No entanto, 23,2\% ( $n=13)$ dos pacientes foram excluídos devido à ausência de dados antropométricos ( $\mathrm{CP}$ e/ou $\mathrm{CB}$ ) e/ou das demais informações pesquisadas. Do total de 43 idosos selecionados, 32,6\% eram do sexo masculino e $67,4 \%$ do sexo feminino, conforme demonstra a Tabela 1. 
Tabela 1 - Distribuição dos idosos, segundo sexo e faixa etária. Município da Grande São Paulo-SP, 2012-2018.

\begin{tabular}{lcccccc}
\hline \multirow{2}{*}{ Faixa Etária } & \multicolumn{9}{c}{ Sexo } \\
\cline { 2 - 7 } & \multicolumn{2}{c}{ Masculino } & \multicolumn{2}{c}{ Feminino } & \multicolumn{2}{c}{ Total } \\
\cline { 2 - 7 } & $\mathbf{n}$ & $\%$ & $\mathbf{n}$ & $\%$ & $\mathbf{n}$ & $\%$ \\
\hline $60-69$ anos & 3 & 42,9 & 4 & 57,1 & 7 & 100 \\
$70-79$ anos & 4 & 28,6 & 10 & 71,4 & 14 & 100 \\
$\geq 80$ anos & 7 & 31,8 & 15 & 68,2 & 22 & 100 \\
\hline Total & 14 & 32,6 & 29 & 67,4 & 43 & 100 \\
\hline
\end{tabular}

Tabela 2 - Via de administração e complicações associadas à TNED. Município da Grande São Paulo-SP, 2012-2018.

\begin{tabular}{lcc}
\hline Variáveis & \multicolumn{2}{c}{ Total } \\
\cline { 2 - 3 } & $\mathbf{n}$ & $\%$ \\
\hline Via de administração & & \\
$\quad$ Nasoenteral & 27 & 62,8 \\
Gastrostomia & 16 & 37,2 \\
Total & 43 & 100,0 \\
\hline Úlcera de pressão & & \\
Presente & 25 & 58,1 \\
Ausente & 18 & 41,9 \\
$\quad$ Total & 43 & 100,0 \\
\hline Diarreia & & \\
Presente & 2 & 4,7 \\
Ausente & 41 & 95,3 \\
$\quad$ Total & 43 & 100,0 \\
\hline Constipação & & \\
Presente & 19 & 44,2 \\
Ausente & 24 & 100,0 \\
$\quad$ Total & 43 & \\
\hline
\end{tabular}

A idade dos idosos variou de 60 a 102 anos, sendo a média de $77,4 \pm 8,5$ anos. Entre as mulheres, a média de idade foi de $78,1 \pm 9,2$ anos e entre os homens, a média foi de 75,9 $\pm 6,9$ anos.

Referente à via de administração da dieta enteral, $62,8 \%$ utilizavam a nasoenteral e $37,2 \%$, a gastrostomia. No que se refere às complicações levantadas, constatou-se que a UP esteve presente em 58,1\% dos idosos, seguida da constipação $(44,2 \%)$ e diarreia $(4,7 \%)$, conforme demonstra a Tabela 2.

Com relação ao estado nutricional, verificou-se que a $\mathrm{CP}$ de $88,4 \%(N=38)$ dos idosos era menor do que $30,5 \mathrm{~cm}$, o que caracteriza depleção de massa muscular. Já a classificação do estado nutricional de acordo com os percentis da CB (Tabela 3), mostrou que 62,9\% estavam com déficit grave de massa (<P10); 4,6\% encontravam-se com déficit leve de massa (P10-P15); 27,9\% estavam com massa normal (P15P75) e 4,6\% estavam com a massa aumentada (P75-P85).

\section{DISCUSSÃO}

No Brasil, estima-se que, a cada ano, há um aumento de cerca de 650 mil novos idosos. Nesta faixa etária, observamse altas prevalências de DCNT e doenças neurológicas, as quais exigem cuidados constantes, medicações ininterruptas e exames periódicos ${ }^{15}$. Tal perfil epidemiológico associa-se a maiores taxas de internações hospitalares, desnutrição, perda de apetite e disfagia, o que, consequentemente, leva a maior necessidade da TNE, seja por via oral ou por sonda ${ }^{16,17}$.

Tabela 3 - Distribuição dos percentis da circunferência do braço, segundo faixa etária e sexo. Município da Grande São Paulo - SP, $2012-2018$.

\begin{tabular}{|c|c|c|c|c|c|}
\hline \multirow{4}{*}{ Faixa etária } & \multicolumn{4}{|c|}{ Homens } & \multirow{3}{*}{ Total } \\
\hline & \multicolumn{4}{|c|}{ Percentis } & \\
\hline & $<\mathrm{P} 10$ & P10 - 15 & P15 - 75 & P75 - 85 & \\
\hline & $\mathrm{n}$ & $\mathrm{n}$ & $\mathrm{n}$ & $n$ & $\mathrm{n}$ \\
\hline 60-69 anos & 3 & - & - & - & 3 \\
\hline 70-79 anos & 4 & - & - & - & 4 \\
\hline$>80$ anos & 5 & 1 & 1 & _ & 7 \\
\hline \multirow[t]{4}{*}{ Subtotal } & 12 & 1 & 1 & - & 14 \\
\hline & $(85,8 \%)$ & $(7,1 \%)$ & $(7,1 \%)$ & & $100,0 \%$ \\
\hline & \multicolumn{4}{|c|}{ Mulheres } & \\
\hline & \multicolumn{4}{|c|}{ Percentis } & Total \\
\hline \multirow[t]{2}{*}{ Faixa etária } & $<\mathrm{P} 10$ & P10 - 15 & P15 - 75 & P75 - 85 & \\
\hline & $n$ & $\mathrm{n}$ & $n$ & $\mathrm{n}$ & $n$ \\
\hline 60-69 anos & 2 & - & 3 & - & 5 \\
\hline 70-79 anos & 7 & - & 2 & 1 & 10 \\
\hline$>80$ anos & 6 & 1 & 6 & 1 & 14 \\
\hline \multirow[t]{2}{*}{ Subtotal } & 15 & 1 & 11 & 2 & 29 \\
\hline & $(51,8 \%)$ & $(3,4 \%)$ & $(37,9 \%)$ & $(6,9 \%)$ & $(100,0 \%)$ \\
\hline \multirow[t]{2}{*}{ Total } & 27 & 2 & 12 & 2 & 43 \\
\hline & $(62,9 \%)$ & $(4,6 \%)$ & $(27,9 \%)$ & $(4,6 \%)$ & $(100,0 \%)$ \\
\hline
\end{tabular}


Na TNE por sonda, a escolha da via de acesso depende da duração prevista para a alimentação enteral, do grau de risco de aspiração ou de deslocamento da sonda, do estado clínico do paciente, da presença ou ausência de digestão e absorção normais e da anatomia do paciente ${ }^{18}$.

No âmbito domiciliar, a TNE é denominada TNED. A indicação da TNED tem aumentando globalmente ${ }^{10}$. Graciano e Ferreti19, ao avaliarem idosos hospitalizados na UTI de um hospital geral particular da região metropolitana da Grande São Paulo, observaram que 81,4\% receberam alta da UTI utilizando nutrição enteral. Estudos sugerem que a TNED proporciona economia dos recursos de saúde, ao gerar maior disponibilidade de leitos nos hospitais, reduz as complicações relacionadas ao ambiente hospitalar, como infecções, e melhoraram o convívio social ${ }^{10,20}$.

O presente estudo, que teve por objetivo realizar um levantamento do estado nutricional e das complicações associadas à TNED em idosos, encontrou que a via de acesso mais utilizada foi a nasoenteral $(62,8 \%)$, seguida da gastrostomia $(37,8 \%)$. Tal resultado é similar ao de outros estudos. Silva e Silveira ${ }^{21}$, ao estudarem pacientes em TNED no município de Alfenas (MG), encontraram que a via de acesso prevalente da nutrição enteral foi a sonda nasoenteral, utilizada por $63 \%$ dos indivíduos, seguida pela sonda nasogástrica (21\%) e gastrostomia (16\%). Azank et al. ${ }^{12}$, ao avaliar adultos atendidos pelo Serviço de Assistência Domiciliar (SAD) de um hospital privado do estado de SP, observaram que $73,3 \%$ receberam alimentação via sonda nasoenteral e $26,6 \%$ via gastrostomia. Martins et al. ${ }^{22}$, ao avaliarem idosos acompanhados pelo serviço de atenção domiciliar de um plano de saúde de Belo Horizonte (MG), encontraram que a via mais utilizada foi a nasoentérica $(59,5 \%)$, seguida da gastrostomia $(40,5 \%)$.

A maior prevalência de sonda nasoenteral, comparada à sonda nasogástrica, pode estar relacionada ao fato de que pacientes em estado crítico podem não tolerar a alimentação diretamente no estômago. Além disso, o risco de pneumonia aspirativa e refluxo gastroesofágico é maior com o uso de sonda nasogástrica. Quando a alimentação enteral é necessária por mais de 3 a 4 semanas, considera-se a realização da gastrotomia ou jejunostomia, pois traz mais conforto ao paciente e evita complicações relacionadas à irritação nasal e do sistema gastrointestinal superior ${ }^{18}$.

Quando analisadas as complicações associadas à TNE, verifica-se na literatura que as mais comuns são infecção na ostomia, deslocamento, vazamento e entupimento da sonda, diarreia, vômito e pneumonia" ${ }^{11}$. No estudo conduzido por Martins et al. ${ }^{22}$, as complicações encontradas foram UP $(43 \%)$, diarreia $(29,4 \%)$, constipação $(19,1 \%)$ e vômitos $(16,2 \%)$. Já no estudo de Silva e Silveira ${ }^{21}$ foi encontrada prevalência de $42 \%$ de diarreia.
No presente estudo, observou-se que as complicações mais prevalentes foram a UP $(58,1 \%)$ e a constipação $(44,2 \%)$. A diarreia esteve presente somente em $4,7 \%$ dos idosos.

As UPs atingem cerca de $37 \%$ dos pacientes acamados ${ }^{23}$. Sabe-se que a desnutrição contribui para o surgimento de alterações físicas, cognitivas e imunológicas, além de contribuir para o surgimento das UP ${ }^{4}$. $O$ envelhecimento constitui outro fator de risco para a formação da UP, pois com o passar dos anos a pele sofre alterações em sua estrutura, apresentando sinais de regressão que se tornam mais destacados por volta dos 65 anos de idade ${ }^{23}$.

A constipação intestinal presente nos pacientes que se alimentam por sonda relaciona-se com a baixa ingestão de água e fibras e o uso de medicamentos ${ }^{18}$. Bittencourt ${ }^{24}$, ao avaliar pacientes em TNE exclusiva internados em hospital geral, encontrou que $70 \%$ deles apresentaram constipação intestinal, sendo que o risco foi de até 7 vezes maior quando a dieta não apresentava fibras. Já a diarreia está relacionada com contaminação bacteriana da fórmula, dieta sem fibra, intolerância à lactose, absorção inadequada de gorduras, intolerância à soja, uso de fármacos, como antibióticos, e cuidados inadequados de higiene e conservação. A incidência da diarreia varia de $2 \%$ a $95 \%$ dos casos, visto que há diferenças na definição e na capacidade de coletar e medir amostras de fezes ${ }^{25}$.

No que se refere aos indicadores clínicos do estado nutricional na população idosa, o presente estudo demonstrou que mais da metade apresentava algum comprometimento do estado nutricional. A depleção de massa muscular, medida por meio da CP, esteve presente em $84 \%$ dos idosos. Já a classificação da CB demonstrou que 62,9\% estavam com déficit grave de massa (percentil <10). Embora dados isolados sejam menos precisos no diagnóstico nutricional, a antropometria, especialmente as medidas de $\mathrm{CB}$ e $\mathrm{CP}$, têm se mostrado um bom indicador do estado nutricional do idoso ${ }^{11}$. A CP é uma medida sensível para avaliar a massa muscular. $O$ ponto de corte de $30,5 \mathrm{~cm}$ proposto por Bonnefoy et al. ${ }^{14}$ mostrou boa correlação com o estado nutricional, tanto em homens quanto mulheres. Já variações da CB podem refletir uma alteração da massa magra, pois ela representa a somatória do tecido ósseo, muscular, gorduroso e epitelial ${ }^{11}$.

O presente estudo apresenta algumas limitações, como amostra pequena e ausência de informações precisas sobre outras características dos idosos avaliados, como tempo da TNED e o tipo de dieta recebida.

\section{CONCLUSÃO}

O levantamento do estado nutricional e das complicações associadas à TNED em idosos permitiu verificar que mais da 
metade apresenta algum déficit de massa muscular e que a UP foi a complicação de maior ocorrência, seguida da constipação e diarreia.

\section{REFERÊNCIAS}

1. World Health Organization. Active ageing: a policy framework. Geneva: World Health Organization; 2002.

2. Malta DC, Moura L, Prado RR, Escalante JC, Schmidt MI, Duncan BB. Mortalidade por doenças crônicas não transmissíveis no Brasil e suas regiões, 2000 a 2011. Epidemiol Serv Saúde. 2014;23(4):599-608.

3. Rizzi L, Rosset I, Roriz-Cruz M. Global epidemiology of dementia: Alzheimer's and vascular types. Biomed Res Int. 2014;2014:908915.

4. Sura L, Madhavan A, Carnaby G, Crary MA. Dysphagia in the elderly: management and nutritional considerations. Clin Interv Aging. 2012;7:287-98.

5. Fideliz MSP, SantanaAFF, Gomes JR. Prevalência de desnutrição hospitalar em idosos. RASBRAN. 2013;5(1):60-8.

6. Brasil. Ministério da Saúde. Agência Nacional de Vigilância Sanitária. Resolução da diretoria colegiada - RDC n ${ }^{\circ} 63$, de 6 de julho de 2000. Brasília: Ministério da Saúde; 2000. [cited 2019 Jul 30]. Disponível em: http://bvsms.saude.gov.br/bvs/ saudelegis/anvisa/2000/rdc0063_06_07_2000.html

7. Brasil. Ministério da Saúde. Informativo técnico sobre a terapia nutricional enteral domiciliar, com foco para a dieta. Brasília: Ministério da Saúde; 2016. [cited 2019 Jul 30]. Disponível em: http://189.28.128.100/dab/docs/portaldab/publicacoes/ info_terapia_nutricional.pdf

8. Foog L. Home enteral feeding part 1: an overview. Br J Community Nurs. 2007;12(6):246-52

9. Costa MP, Sturtz G, Costa FPP, Ferreira MC, Barros Filho TEP. Epidemiologia e tratamento das úlceras de pressão: experiência de 77 casos. Acta Ortop Bras. 2005;13(3):124-33.

10. Ojo O. The challenges of home enteral tube feeding: a global perspective. Nutrients. 2015;7:2524-38.

11. Vitolo MR. Parte IX - Idoso. In: Nutrição: da gestação ao envelhecimento. Rio de Janeiro: Rubio; 2008. p.393-410.
12. Azank AT, Leandro-Merhi VA, Polisseli C, Oliveira MRM. Indicadores nutricionais em pacientes alimentados por sonda, em sistema de "home care". Arq Catarinenses Med. 2009;38(4):11-8.

13. Kuczmarski MF, Kuczmarski RJ, Najjar M. Descriptive anthropometric reference data for older Americans. J Am Diet Assoc. 2000;100(1):59-66.

14. Bonnefoy M, Jauffret M, Kostka T, Jusot JF. Usefulness of call circumference measurement in assessing the nutritional state of hospitalized elderly people. Gerontology. 2002;48(3):162-9.

15. Veras R. Envelhecimento populacional contemporâneo: demandas, desafios e inovações. Rev Saúde Pública. 2009;43(3):548-54.

16. Posthauer ME, Dorner B, Friedrich EK. Enteral nutrition for older adults in healthcare communities. Nutr Clin Pract. 2014;29(4):445-8.

17. Fragas RFM, Oliveira MC. Risk factors associated with malnutrition in hospitalized patients. Rev Nutr. 2016;29(3):329-36.

18. Raymond JL, Ireton-Jones CS. Administração de alimentos e nutrientes: métodos de terapia nutricional. In: Mahan LK, Raymond JL, eds. Krause: alimentos, nutrição e dietoterapia. $13^{\mathrm{a}}$ ed. Rio de Janeiro: Saunders Elsevier; 2013. p.658-75.

19. Graciano RDM, Ferretti REL. Nutrição enteral em idosos na unidade de terapia intensiva: prevalência e fatores associados. Geriatria \& Gerontologia. 2008;2(4):151-5.

20. Zaban ALRS. Nutrição enteral domiciliar: um novo modelo de gestão econômica do Sistema Único de Saúde [Dissertação de Mestrado]. Brasília: Universidade de Brasília; 2009.

21. SilvaAC, Silveira SA.Perfilepidemiológico enutricional de usuários de nutrição enteral domiciliar. Demetra. 2014;9(3):783-94.

22. Martins AS, Rezende NA, Torres HOG. Sobrevida e complicações em idosos com doenças neurológicas em nutrição enteral. Rev Assoc Med Bras. 2012;58(6):691-7.

23. Dealey C. Cuidando de feridas: um guia para enfermeiras. $3^{\mathrm{a}}$ ed. São Paulo: Atheneu; 2008.

24. Bittencourt AF. Diarreia e constipação intestinal em terapia nutricional enteral [Dissertação de Mestrado]. São Paulo: Universidade de São Paulo; 2013. 117p.

25. Chang SJ, Huang HH. Diarrhea in enterally fed patients: blame the diet? Curr Opin Clin Nutr Metab Care. 2013;16(5):588-94.

Local de realização do estudo: Universidade Paulista (UNIP), Alphaville, SP, Brasil.

Conflito de interesse: Os autores declaram não haver. 